\title{
Therapeutic hypertension system based on a microbreathing pressure sensor system
}

This article was published in the following Dove Press journal:

Medical Devices: Evidence and Research

I3 May 201 I

Number of times this article has been viewed

Ziji Diao'

Hongying Liu'

Lan Zhu'

Xiaoqiang Gao'

Suwen Zhao'

Xitian $\mathrm{Pi}^{1,2}$

Xiaolin Zheng ${ }^{1,2}$

'Key Laboratory of Biorheological Science and Technology, Chongqing University, Ministry of Education, Chongqing; ${ }^{2}$ Key Laboratories for National Defense Science and Technology of Innovative Micronano Devices and System Technology,

Chongqing, People's Republic of China

Correspondence: Hongying Liu College of Bioengineering, Chongqing University, Chongqing, 400030, People's Republic of China

$\mathrm{Tel}+8602365$ I I 193।

Fax +86023 65II | 93 I

Email liuhongying@cqu.edu.cn;

diaoziji@I26.com
Background and methods: A novel therapeutic system for the treatment of hypertension was developed on the basis of a slow-breath training mechanism, using a microbreathing pressure sensor device for the detection of human respiratory signals attached to the abdomen. The system utilizes a single-chip AT89C51 microcomputer as a core processor, programmed by Microsoft Visual $\mathrm{C}++6.0$ to communicate with a PC via a full-speed PDIUSBD12 interface chip. The programming is based on a slow-breath guided algorithm in which the respiratory signal serves as a physiological feedback parameter. Inhalation and exhalation by the subject is guided by music signals.

Results and conclusion: Our study indicates that this microbreathing sensor system may assist in slow-breath training and may help to decrease blood pressure.

Keywords: hypertension, microbreathing sensor, single-chip microcomputer, slow-pace breathing

\section{Introduction}

Hypertension is an important cause of cardiovascular disease and, at present, the treatment of hypertension worldwide is mainly via drug therapy. However, due to the expense of long-term medication, as well as complicated regimens, it is often difficult for patients to be compliant with therapy. Furthermore, many antihypertensive drugs have side effects, including depression, angioneurotic edema, and disordered sleep. The shortcomings of drug therapy have prompted researchers to explore drug-free strategies to treat hypertension. Researchers have shown that blood pressure can be lowered by improving lifestyle, eg, increasing exercise, reducing salt intake, and controlling weight. ${ }^{1,2}$ Physical relaxation training, eg, yoga and meditation, are also able to reduce blood pressure. ${ }^{3,4}$ However, patient behavior largely determines the success of nonpharmacological therapy, so therapeutic lifestyle modalities cannot be regarded as systematic and scientific.

In recent years, an increasing number of studies have shown that blood pressure can be lowered by decreasing respiratory rate, ie, by training patients to breathe more slowly and regularly. ${ }^{5,6,10} \mathrm{~A}$ breath-training instrument has been devised to help hypertensive patients to reduce their respiration rate to less than 10 breaths per minute. Clinical research indicates that use of this instrument for 15 minutes daily over 8 weeks can achieve a significant decrease in blood pressure. ${ }^{7}$ However, the functioning of this instrument is so simple that, although it can monitor respiratory rate, it cannot store data for the training process, replay the recording, or perform data and statistical analysis. It also does not provide any information on changes in 
blood pressure related to breathing ratio and respiration rate, so researchers are not able to investigate the regulatory mechanism involved in respiratory training. Therefore, in this study, we used biofeedback technology, sensor technology, and electronic circuit theory to design a treatment system that could train patients with hypertension to perform quantified slow breathing exercises, and to investigate the mechanism of respiratory training, and its value in the treatment of hypertension.

\section{Methods}

\section{System design}

Figure 1 shows a diagram of the system, which includes both hardware and software components. The hardware comprises an integrated LM324 amplifier chip (Yitaike Co Ltd, Beijing, China), an AT89C51 microcontroller (Atmel, San Jose, CA), a TLC2543 A/D converter chip (Texas Instruments, Salt Lake City, UT), and a PDIUSBD12 USB interface chip (Philips, Amsterdam, Netherland). Breathing signals detected by the breathing sensors are amplified and filtered through the circuits, and then the analog signals are converted into digital signals which are transmitted to the computer via a USB data acquisition module for further processing.

The software exploits the bioinformation feedback principle, interpreting the respiratory signal as a feedback unit, uses VC++6.0 (Microsoft, Redmond, WA) to design the guiding algorithm, and also uses different music rhythms (auditory signals) and progress bars (visual signals) to guide patients in a cyclic pattern of continuously adjusting and reducing their respiratory rate. At the same time, the system stores respiratory signals during the training process. The signal is analyzed to calculate the changing respiratory ratio and to investigate the regulatory mechanisms involved in the effect of respiratory training on blood pressure.

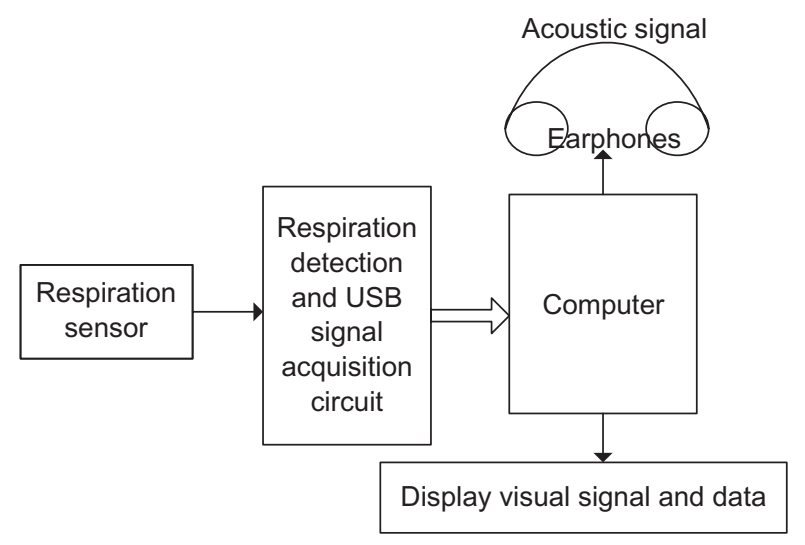

Figure I Block diagram of the system.

\section{Hardware circuit design}

\section{Respiration signal detection circuit}

YXF-2 piezoresistive microathletic-type breathing sensors (Beijing Yi Song Technology Co Ltd, Beijing, China) were used to acquire signals. The range of the sensor is $0-300 \mathrm{~g}$, and the full output range is $>30 \mathrm{mV} / 300 \mathrm{~g} / 5 \mathrm{~V}$ (direct current). Its working principle is that when the microbreathing sensor is attached to the chest/abdomen, fluctuating movements caused by breathing will change the resistance value of the piezoresistive sensor, lead to an imbalance at the bridge, putting out the corresponding voltage signal. Because the fluctuating movement of the chest/abdomen is periodic, changes in resistance and output voltage signal from the bridge should follow a similar fluctuating pattern. A standard respiratory signal is obtained via amplification and filtering. The detection circuit for this device is shown in Figure 2.

The respiratory signal detection circuit consists of three parts, ie, a preamplifier, a band-pass filter circuit, and a secondary amplifier circuit. The sensor's output consists of dozens of $\mathrm{mV}$, while the analog input voltage range for the TLC2543 A/D converter chip covers the range of $0 \mathrm{~V}$ to $+5 \mathrm{~V}$. In order to make these two ranges match as closely as possible, the preamplification is set to be about $10 \times$ and the post-inverting amplifier is set to about $20 \times$, and thus the final output voltage (Uo) can meet the basic input requirement of the TLC2543 converter chip. The human

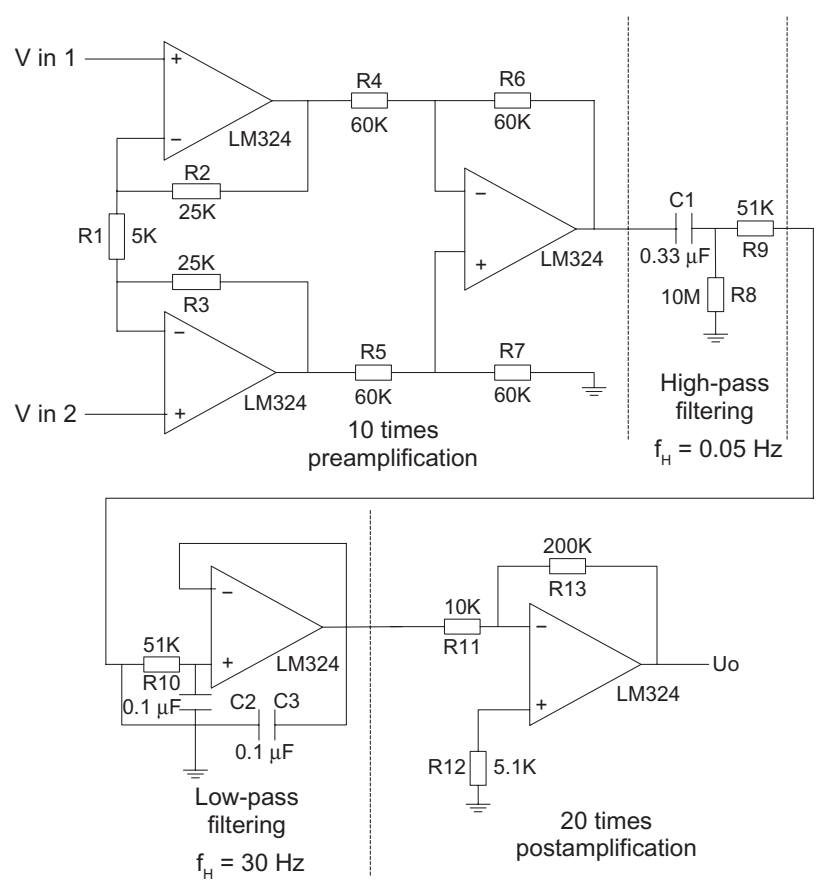

Figure 2 Breathing signal detection circuit. 
respiratory rhythm is generally reasonably stable, with a corresponding frequency range of about $0.125-2.5 \mathrm{~Hz}$, so a high-pass filter comprising a $0.33 \mu \mathrm{F}$ capacitor and a $10 \mathrm{~m} \Omega$ resistor is added after the first amplifier. The time constant, $\tau$, of the high-pass filter is about 3.3 seconds, and the cutoff frequency is $0.05 \mathrm{~Hz}$. The cutoff frequency of the low-pass filter connected after the high-pass filter is $30 \mathrm{~Hz}$. Therefore, the bandwidth of the band-pass filter is $0.05-30 \mathrm{~Hz}$.

\section{A/D converter circuit}

The advantages of the 12-bit serial analog-digital TLC2543 conversion chip with 11 input ends are a fast converting speed, good stability, and a simple microprocessor interface. Considering the superior serial communication function of the AT89C51 microcontroller, its bit manipulation capability, and its simplicity when connected with the serial A/D conversion chip, the serial A/D conversion chip was able to be combined with the AT89C51 microcontroller to form a data acquisition system. There are two possible approaches in the development of such a data acquisition system. One is to use a serial input and output interface based on byte operation, and the other is to use common input and output wires based on bit operations. The requirements of these methods are different, eg, the switching speed of the A/D converter chip, the operation mode, and the Baud rate of data transmission. The second approach was used for this study. Figure 3 shows the interface circuit diagram for the TLC2543 and the AT89C51 microcontroller. AIN0, the TLC2543 analog input channel, is selected as the analog input end and connected with the output (Uo) of the preamplifier circuit. The reference voltage (REF+) and TLC2543 are connected with the power AT89C51 at $+5 \mathrm{~V}$, and the REF was connected to the GND. On the chip selection terminal, when CS_N is

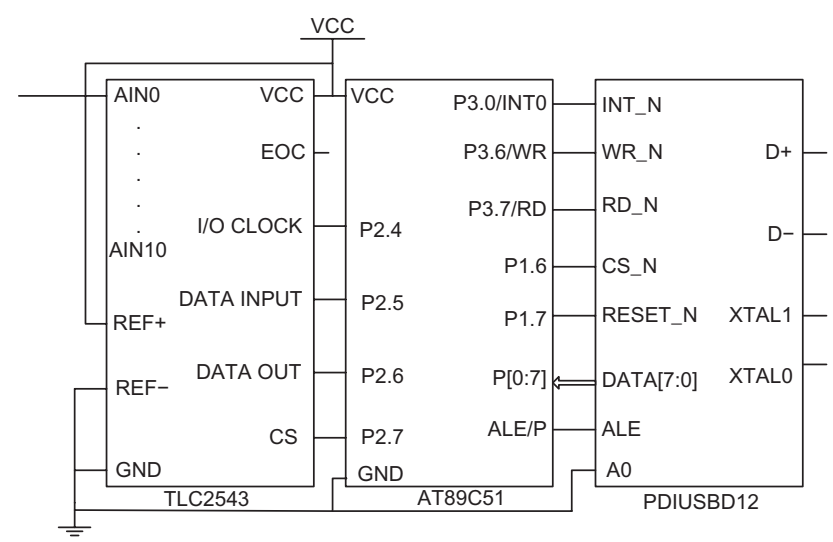

Figure 3 Circuit schematic of USB interface based on PDIUSBDI 2 and A/D conversion based on TLC2543. at the value of 1 the $\mathrm{I} / \mathrm{O}$ clock and data input are disabled, otherwise the I/O clock and data input are enabled. The data input is used to input the chip's control word, and the 8-bit control data are serially fed to the TLC2543 through the P2.5 pin of the AT89C51 microcontroller. The higher four data entries determine the number of the analog channel that needs conversion, while the lower four entries determine the length and polarity (ie, unipolar or bipolar) of the converted output data.

\section{USB interface circuit}

Figure 3 shows the interface circuit of the PDIUSBD12 and the AT89C51 microcontroller. The P0 pin is connected to the DATA0-DATA7 pin of the PDIUSBD12 to transmit 8-bit data. The CS_N is connected with the P1.6 pin; when $\mathrm{P} 1.6=0$, the PDIUSBD12 is enabled, and when P1.6 = 1 , the PDIUSBD12 is disabled. Whether CS_N is enabled or not, the order by which the data will be input to the PDIUSBD12 is determined by the level of A0. When A0 is at a low level, the multiple address/data bus mode controlled by the ALE is selected. The $6 \mathrm{M}$ external crystal is used directly as the source of the clock circuit ${ }^{8}$ for the PDIUSBD12. The D+ and D- of the USB uplink interfaces ${ }^{9}$ are brought down to a low level by a $1 \mathrm{~m} \Omega$ resistor.

\section{Software design}

The software design comprises two parts. One is the program for the USB device (ie, USB bottom firmware). This program is mainly used to complete the tasks of the USB device, ie, enumerating, protocol processing, and data exchanging. The other is the program design of the PC, including the USBdriver program and customer service program. The USB bottom firmware and driver program were provided by the ZHOU Ligong PDIUSBD12 Smart development board, and there are only slight corresponding changes based on the above mentioned programs. Therefore, the description of the USB firmware and driver design is omitted here.

The core design of the user service program is the algorithm for recognition of the respiratory signal and the music rhythm used to guide respiratory rhythm. The program has been developed with $\mathrm{VC}++6.0$ and relies on EasyUSB.DLL API. ${ }^{8}$

\section{Feature point recognition of respiratory signal}

The periodic respiratory signal contains peaks and valleys, where the valleys indicate the beginning of inhalation and the peaks indicate the beginning of exhalation. The ascending 
branch duration of the waveform represents inhalation time, while the descending branch duration represents exhalation time. Obtaining accurate respiratory signal peaks and valleys, as well as calculating the inhalation time and exhalation time, are important prerequisites for generating the musical rhythm guiding the respiratory rhythm. In this study, a method of computing the difference point by point is used to detect respiratory signal peaks and valleys in order to calculate the length of time for inhalation and exhalation. The respiration rate and respiratory ratio (exhalation time:inhalation time) are calculated from these figures. Figure 4 shows the real-time detection of respiratory signal peaks and valleys, as well as the changing trend of respiration rate and respiration ratio.

\section{Algorithm for the music rhythm}

Blood pressure can be decreased by the following steps. First, gradually lengthen the subject's exhalation time and leave the inhalation time unchanged, so as to decrease the respiratory rate slowly until the minimum respiratory rate that the subject can tolerate is reached. Second, keep the minimum respiratory rate (while maintaining the same respiratory ratio) for 5 minutes in the breathing exercise. Third, after the respiratory training, the subject was required to sit quietly for 1 minute. Then, the subject's blood pressure was measured, and systolic and diastolic values were found to decrease. Accordingly, we designed an algorithm to generate breathing exercises guided by a musical rhythm, which consists of music corresponding to exhalation and inhalation. The subject's breathing signal serves as a feedback quantity to form a physiological signal feedback loop. The subject's respiratory rate correspondingly decreases as the exhalation time lengthens. The progress bar provided by $\mathrm{VC}++6.0$ can guide the subject to slow down his/her breathing. Its state is synchronized with the tempo of the music. To guide slow breathing, subjects may select the
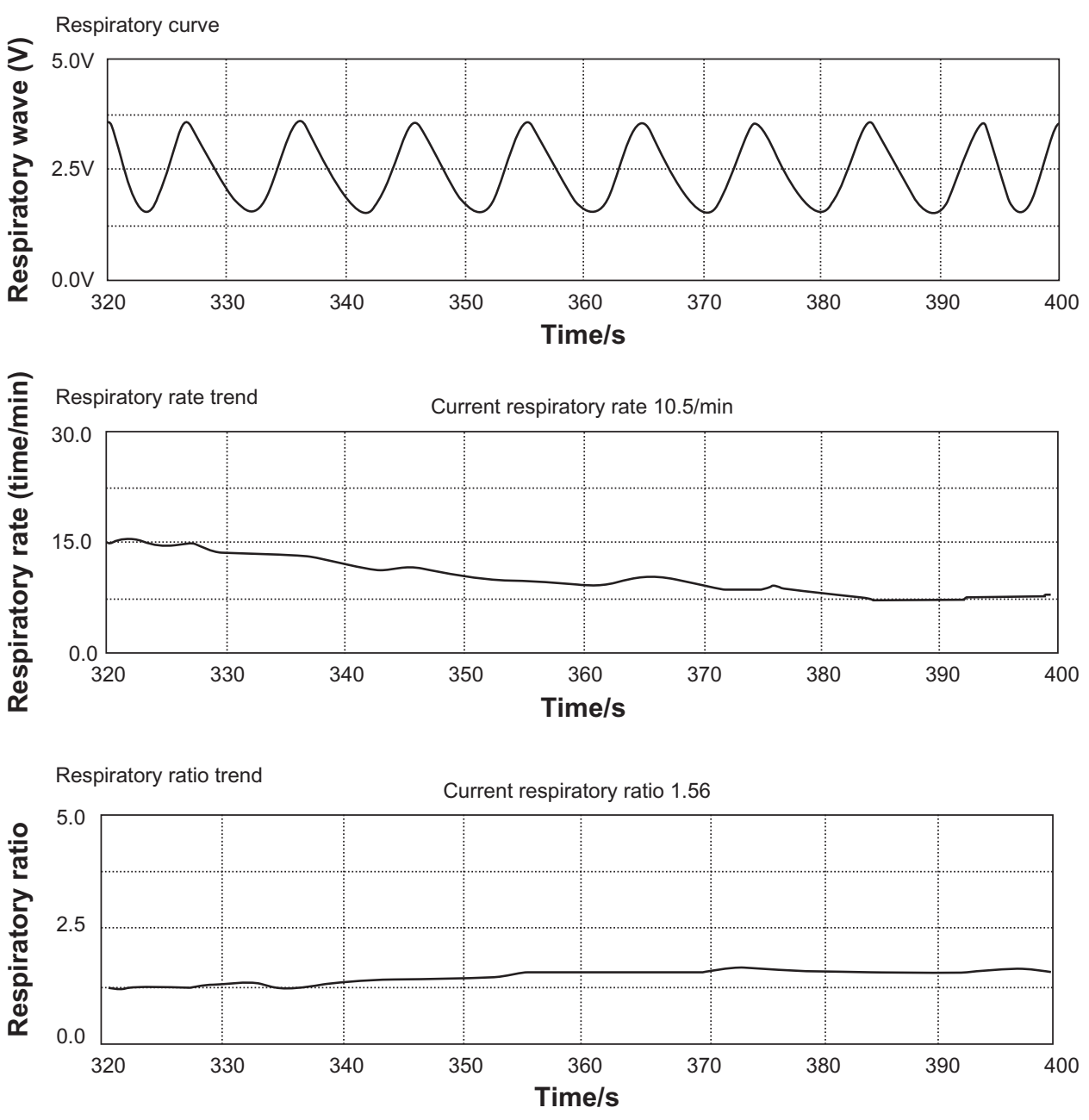

Figure 4 Respiratory trace trend graphs of breaths per minute and ratio of expiration time to inspiration time. 


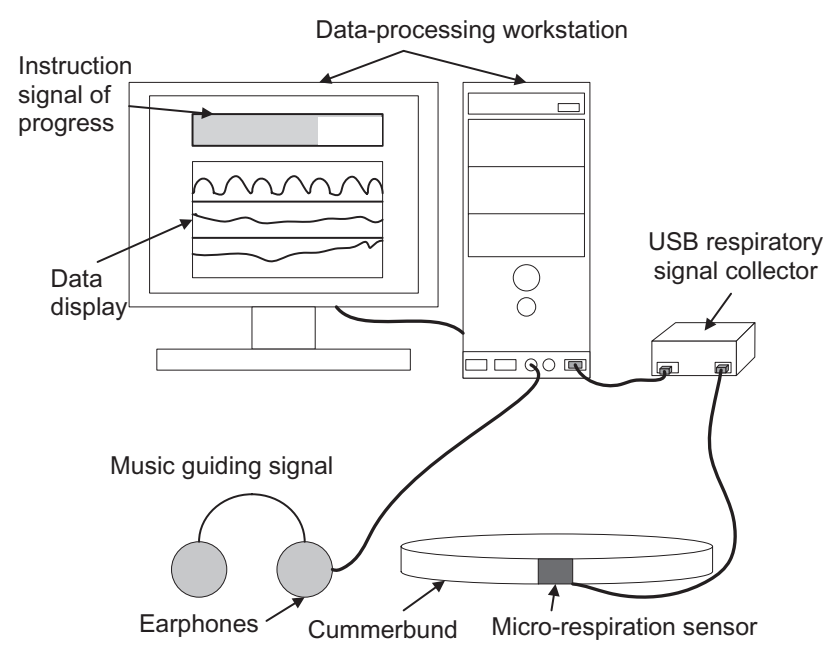

Figure 5 Experimental frame of the hypertension therapeutic system.

progress bar or the guiding music rhythm only or both. Figure 5 shows the experimental system block diagram of slow breath training for the treatment of hypertension. The subjects follow the auditory and visual signals to guide respiratory movement.

\section{Preliminary study using the system}

The main purpose of our study was to verify whether this system has a blood pressure-lowering effect. The study population included 32 hypertensive subjects recruited from the community, comprising 20 men and 12 women, aged $35-72$ years and weighing $52-88 \mathrm{~kg}$. Thirteen were receiving antihypertensive drugs, with no dose change for 1 week before the study (Table 1). Exclusion criteria included concomitant angina, heart failure, renal failure, cerebrovascular disease, pregnancy, blindness, deafness, and inability to use a digital blood pressure monitor. Patients with blood pressure classified as Stage III (systolic blood pressure $\geq 180 \mathrm{mmHg}$ or diastolic blood pressure $\geq 110 \mathrm{mmHg})^{11}$ were excluded.

The experiment began at 9 am every day (with a suitable breakfast, but no coffee, tea, or similar drinks) and lasted for 42 days. After the subjects had been sitting quietly for 10 minutes, their blood pressure was measured and recorded. The respiratory training system was then used to

Table I Patient demographic data

\begin{tabular}{llll}
\hline Age (years) & $\begin{array}{l}\text { Malel } \\
\text { female }\end{array}$ & $\begin{array}{l}\text { Medicated/ } \\
\text { nonmedicated }\end{array}$ & Weight (kg) \\
\hline $35-72$ & $20 / 12$ & $19 / 13$ & $52-94$ \\
\hline
\end{tabular}

guide them to breathe. After each training session, blood pressure was recorded. In addition, they were required to monitor their blood pressure at home, then bring back the data the following day. Mean post-training blood pressure was calculated. Figures $6 \mathrm{~A}$ and $6 \mathrm{~B}$ shows the average systolic and diastolic blood pressure in the 32 subjects before and after training. The two sets of data (average systolic and diastolic blood pressure before and after experiment) were analyzed using the $t$-test function in Excel (Microsoft, Redmond, WA) to find out whether there was any significant difference in average blood pressure levels before and after training.

\section{Results}

Both systolic and diastolic blood pressure decreased significantly before and after the experiment and was most marked when the respiratory rate was below eight breaths per minute. The lowest average respiratory rates that eight subjects were able to achieve decreased gradually as the experiment went on. This indicates the subjects were able to adapt to the designed training system. Figure 7 shows the decrease in systolic and diastolic blood pressure, with an average overall reduction of $-7.3 \mathrm{mmHg}$ in systolic blood pressure and $-4.9 \mathrm{mmHg}$ in diastolic blood pressure $(P<0.001)$.

\section{Discussion}

There are some limitations in the design of this experiment, ie, its relatively short duration which was insufficient to confirm that the system has a long-term blood pressure-lowering effect, inclusion of some subjects exercising good daily lifestyle measures (including low-salt diet, weight control, exercise) which may have influenced our preliminary data, and it is not clear whether we could achieve the best effect on lowering blood pressure when the respiration rate is at the tolerable limit for the subjects, ie, whether there is another optimal respiratory rate threshold. All these issues need to be further researched.

These newly developed slow breath training methods for the treatment of hypertension have already been shown to have a dramatic effect on blood pressure, but because of methodological differences in the studies done to date, researchers hold different opinions regarding the mechanism by which slow breathing regulates the cardiovascular system. Therefore, we plan on using this slow-breathing training system to further investigate the relationship between the respiratory and cardiovascular systems. 

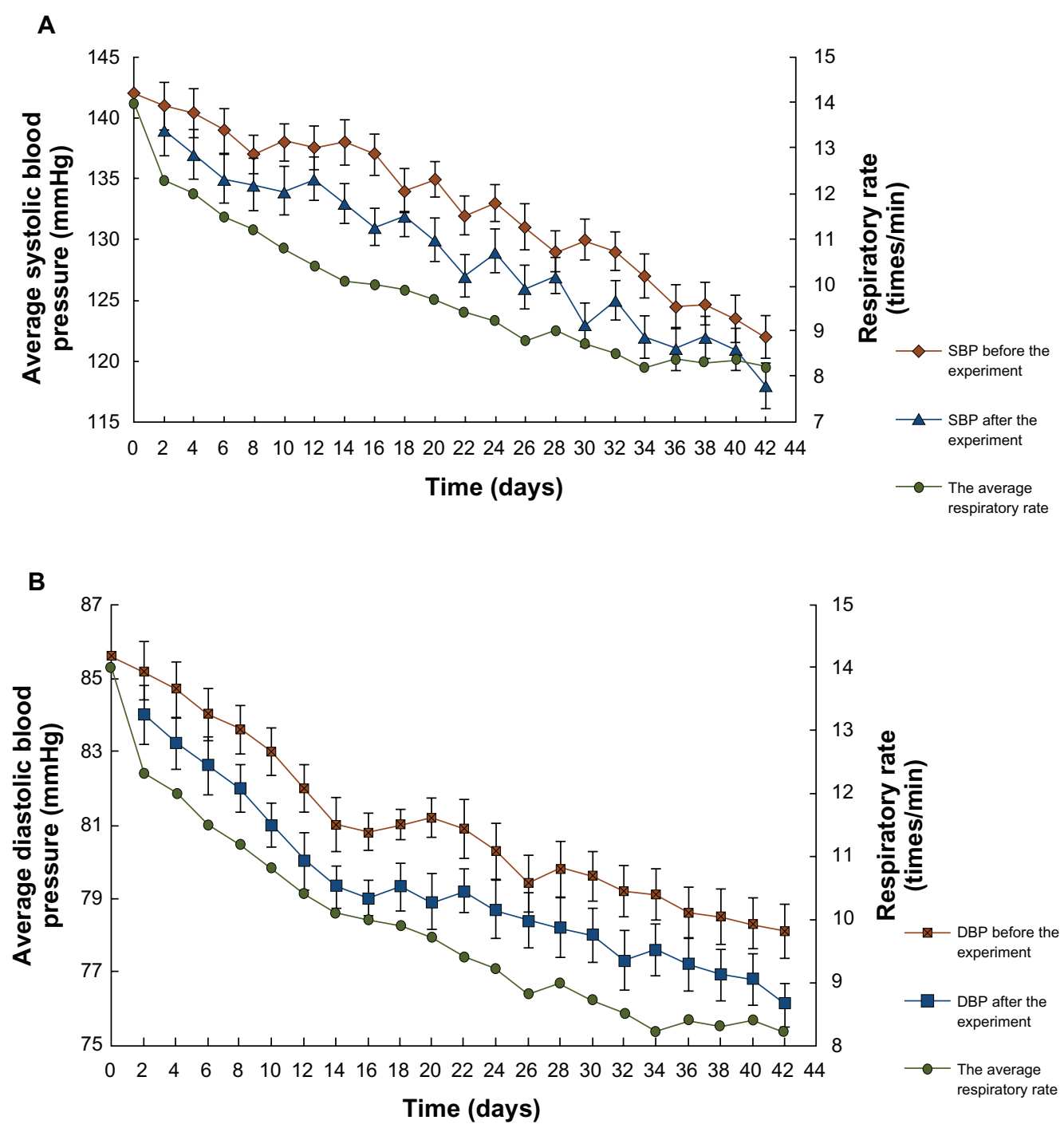

Figure 6 Change between pre- and post-experimental blood pressure and the trend of mean minimum respiratory rate in a typical experiment. A) Change in pre- and post-average systolic blood pressure and the trend of mean minimum respiratory rate in a typical experiment. B) The change between pre-and post-average diastolic blood pressure and the trend of mean minimum respiratory rate in a typical experiment.

Time (days)

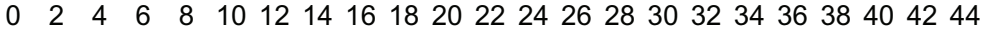

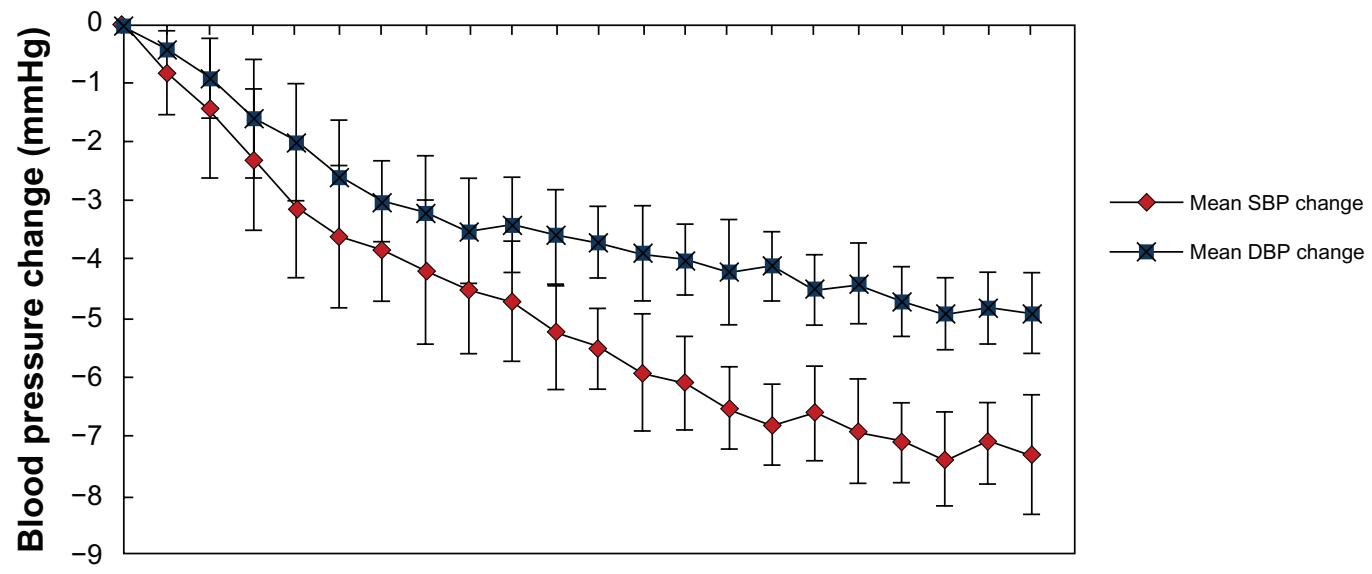

Figure 7 Mean systolic and diastolic blood pressure changes during the 6-week experiment. 


\section{Acknowledgments}

This project was supported by the National Eleventh-Five Year Research Program of China, the Programs for Science and Technology Development of CSTC, and the Fundamental Research Funds for the Central University.

\section{Disclosure}

The authors report no conflicts of interest in this work.

\section{References}

1. Kikuo A. Non-pharmacologic measures for lowering blood pressure. Cardiovasc Drugs Ther. 1989;3:847-852.

2. American Heart Association. Nonpharmacological approaches to the control of high blood pressure. Final report of the Subcommittee on Nonpharmacological Therapy of the 1984 Joint National Committee on Detection, Evaluation, and Treatment of High Blood Pressure. Hypertension. 1986;8:444-467.

3. Brownstein AH, Dembert ML. Treatment of essential hypertension with yoga relaxation therapy in a USAF aviator: A case report. Aviat Space Environ Med. 1989;60:684-687.
4. Blackwell B. Transcendental meditation in hypertension. Individual response patterns. Lancet. 1976;1:223-226.

5. Bernardi L, Gabutti A, Porta C, et al. Slow breathing reduces chemoreflex response to hypoxia and hypercapnia, and increases baroreflex sensitivity. J Hypertens. 2001;19:2221-2229.

6. Bernardi L, Porta C, Spicuzza L, et al. Slow breathing increases arterial baroreflex sensitivity in patients with chronic heart failure. Circulation. 2002;105:143-145.

7. Grossman E, Grossman A, Schein MH, et al. Breathing control lowers blood pressure. J Hum Hypertens. 2001;15:263-269.

8. Shuo Wang, Gang Li. The PDIUSBD12 interface device application design of USB. Journal of Computer Applications. 2002;4:56-59.

9. Ligong Z. PDIUSBD12 USB for Firmware Programming and Program Development. Beijing, China: Beijing Aerospace University Press; 2003.

10. Almeida P, Guilherme Mariano Pego, et al. Treatment of resistant hypertension by device-guided breathing. Am J Hypertens. 2003;16: 333-334.

11. Cushman WC, Cohen JD, Jones RP, Marbury TC, Rhoades RB, Smith LK. Comparison of the fixed combination of enalapril/diltiazem ER and their monotherapies in stage 1 to 3 essential hypertension. $A m J$ Hypertens. 1998;11:23-30.
Medical Devices: Evidence and Research

\section{Publish your work in this journal}

Medical Devices: Evidence and Research is an international, peerreviewed, open access journal that focuses on the evidence, technology, research, and expert opinion supporting the use and application of medical devices in the diagnosis, treatment and management of clinical conditions and physiological processes. The identification of novel

\section{Dovepress}

devices and optimal use of existing devices which will lead to improved clinical outcomes and more effective patient management and safety is a key feature. The manuscript management system is completely online and includes a quick and fair peer-review system. Visit http://www. dovepress.com/testimonials.php to read real quotes from authors.

\footnotetext{
Submit your manuscript here: http://www.dovepress.com/medical-devices-evidence-and-research-journal
} 